\title{
Bioimpedância elétrica e sua aplicação em avaliação nutricional
}

\author{
Bioelectric impedance analysis and its \\ use for nutritional assessments
}

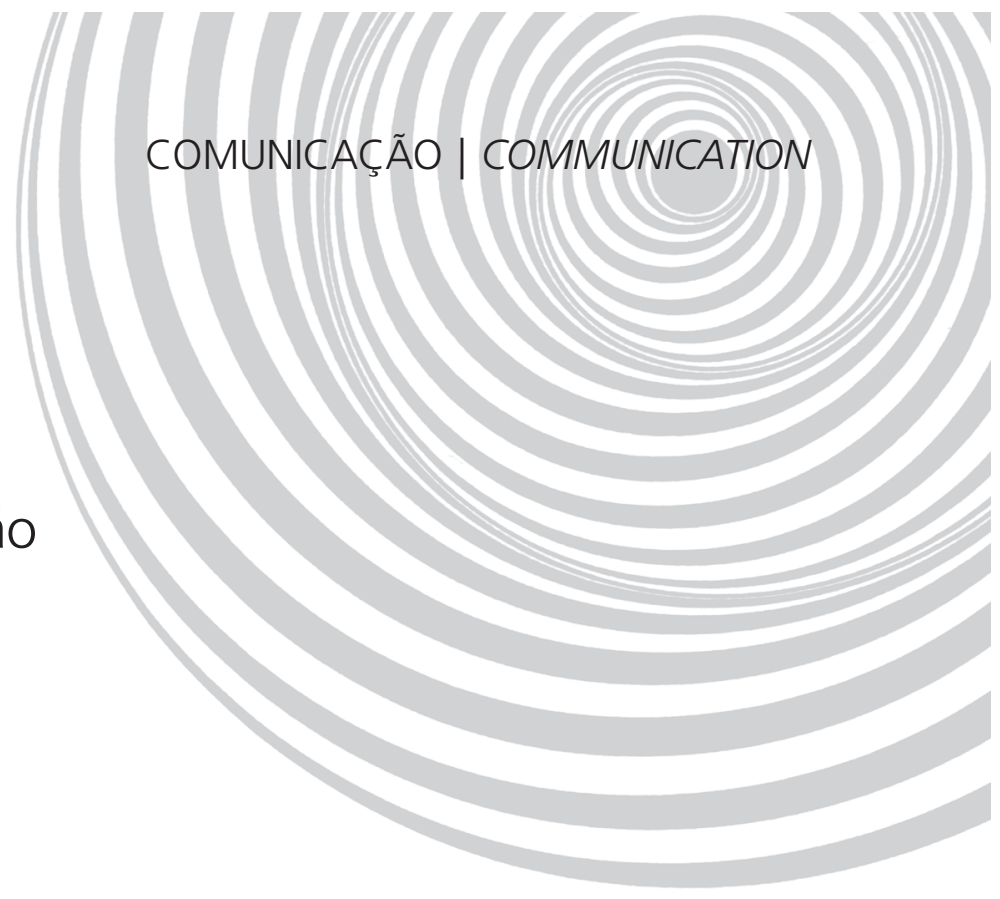

Michaela EICKEMBERG'

Carolina Cunha de OLIVEIRA'

Anna Karla Carneiro RORIZ ${ }^{1}$

Lílian Ramos SAMPAIO'

RE S U M O

A busca por métodos de estimativa da composição corporal é uma preocupação constante da comunidade científica, com vistas à obtenção de um acurado diagnóstico do estado nutricional de indivíduos e populações. A bioimpedância elétrica tem sido uma alternativa atraente na avaliação da composição corporal, pela possibilidade de se trabalhar com equipamento não invasivo, portátil, de fácil manuseio, boa reprodutibilidade e, portanto, viável para a prática clínica e para estudos epidemiológicos. Sua utilização, que tem como finalidade determinar o fracionamento da composição corporal, tem sido apontada como uma técnica capaz de superar alguns desafios encontrados em outros métodos para avaliar o estado nutricional. Entre os componentes da bioimpedância elétrica, o ângulo de fase consiste em uma ferramenta cada vez mais utilizada na prática clínica, sendo estudado como indicador prognóstico e de estado nutricional. Esse ângulo indica alterações na composição corporal e na função da membrana celular, portanto, no estado de saúde de indivíduos. Dada a carência de estudos brasileiros sobre determinadas aplicações da bioimpedância elétrica, a proposta deste estudo, buscando contribuir com a literatura, é traçar um panorama sobre o emprego dessa técnica e, ainda, apresentar trabalhos que a comparam com outros métodos de avaliação nutricional e composição corporal.

Termos de indexação: Avaliação nutricional. Composição corporal. Impedância elétrica.

A B S T R A C T

The scientific community is always searching for methods that estimate body composition because of the importance of making accurate nutritional status diagnoses of individuals and populations. Bioelectrical impedance analysis Bioelectrical impedance is an attractive alternative for determining body composition because it is noninvasive, portable, easy to handle and has good reproducibility, and therefore, is viable for clinical practice and epidemiological studies. Bioelectrical impedance whose purpose is the determination of body composition, has been pointed out as capable of overcoming some challenges present in other methods that assess nutritional status. Among Bioelectrical impedance components, the use of phase angle in clinical practice

\footnotetext{
1 Universidade Federal da Bahia, Escola de Nutrição. Av. Araújo Pinho, 32, Canela, 40110-150, Salvador, BA, Brasil. Correspondência para/Correspondence to: M. EICKEMBERG. E-mail: <mieickemberg@yahoo.com.br>.
} 
884 M. EICKEMBERG et al.

has been increasing, since it is used as an indicator of prognosis and nutritional status. This angle detects changes in body composition and cell membrane function, hence, in health status. In Brazil, there is a scarcity of studies on some Bioelectrical impedance applications. In order to contribute to the available literature, this study aimed to draw an overview about the use of this technique and present studies that compare Bioelectrical impedance with other nutritional and body composition assessment methods.

Indexing terms: Nutritional assessment. Body composition. Eletric impedance.

\section{N T R O D U ÇÃ O}

O interesse pela composição corporal vem crescendo consideravelmente nos últimos anos, pois não há dúvidas sobre a relação do aumento da gordura corporal e sua distribuição com desordens metabólicas e doenças cardiovasculares, a exemplo da diabetes, hipertensão arterial e dislipidemias $^{1,2}$.

Dentre os métodos utilizados para a avaliação da composição corporal, a Bioimpedância Elétrica (BIA) tem sido amplamente utilizada, sobretudo pela alta velocidade no processamento das informações, por ser um método não invasivo, prático, reprodutível e relativamente barato, que estima, além dos componentes corporais, a distribuição dos fluidos nos espaços intra e extracelulares, bem como a qualidade, tamanho e integridade celular ${ }^{3-6}$.

A propriedade elétrica dos tecidos tem sido estudada desde 1871, mas somente em 1970 os fundamentos da BIA foram descritos ${ }^{3,4}$. Os primeiros estudos com BIA ocorreram nas décadas de 1930 e 1940, relacionando a impedância com o fluxo sanguíneo ${ }^{7,8}$. Posteriormente, Thomasset ${ }^{9}$ e Hoffer et al. ${ }^{10}$ estudaram a relação da impedância com a água corporal total e a massa magra.

Atualmente, a BIA tem sido validada para estimar a composição corporal e o estado nutricional de indivíduos saudáveis, e em diversas situações clínicas ${ }^{6,11-13}$, como desnutrição, traumas, câncer, pré e pós-operatório, hepatopatias, insuficiência renal, gestação, bem como em crianças, idosos e atletas.

O objetivo deste artigo é descrever e discutir os princípios e a utilização da BIA, além de apresentar estudos que comparam esse método com outros de avaliação nutricional e de composição corporal.

\section{Princípios da bioimpedância}

Para avaliação da composição corporal, a técnica da BIA baseia-se no modelo de um condutor cilíndrico, com comprimento e área transversal uniformes e homogêneos, ao qual o corpo humano se assemelha. Tal comparação é questionável, pois a composição corporal é heterogênea. Assume-se então, que o corpo humano seja composto por cinco cilindros conectados em série, e não por um único cilindro ${ }^{3,4}$.

Segundo a literatura, o volume do cilindro, assim como do corpo humano, é diretamente relacionado com a impedância total do corpo, estimando-se a composição corporal através da estatura ao quadrado, dividida pela resistência $(V=$ estatura $2 / R)$. A impedância à corrente através do corpo é relacionada diretamente ao comprimento do condutor e inversamente à sua área transversal ${ }^{3,4}$.

A BIA fundamenta-se no princípio de que os tecidos corporais oferecem diferentes oposições à passagem da corrente elétrica. Essa oposição, chamada impedância (Z), tem dois vetores, denominados Resistência (R) e Reactância $(X C)^{3}$.

Em sistemas biológicos, a corrente elétrica é transmitida pelos íons diluídos nos fluidos corporais, especificamente íons de sódio e potássio. Os tecidos magros são altamente condutores de corrente elétrica devido à grande quantidade de água e eletrólitos, ou seja, apresentam baixa resistência à passagem da corrente elétrica. Por outro lado, a gordura, o osso e a pele constituem um meio de baixa condutividade, apresentando, portanto, elevada resistência ${ }^{3,4,14}$.

O vetor $\mathrm{R}$ mede a oposição ao fluxo da corrente elétrica através dos meios intra e extracelulares do corpo, estando diretamente associado 
ao nível de hidratação desses meios. O vetor Xc mede a oposição ao fluxo da corrente causada pela capacitância produzida pela membrana celular 3,4,15.

Também chamada de Resistência Capacitiva, a Xc significa a oposição do fluxo elétrico, causada pela capacitância (propriedade de armazenar energia elétrica sob a forma de um campo eletrostático). Um capacitor é formado por duas ou mais membranas condutoras, separadas por um material isolante ou não condutivo, capaz de armazenar energia elétrica. A membrana citoplasmática do ser humano é constituída por duas camadas de material proteico (bom condutor) e uma camada de lipídeo (isolante). Dessa forma, a membrana celular atua como se fosse um capacitor, oferecendo Xc (capacitância) ${ }^{3,4}$.

Assim, no corpo humano, as membranas celulares podem armazenar a energia por um pequeno período de tempo, "atrasando" a corrente. Esse "atraso" no fluxo da corrente elétrica, causado pela capacitância, gera uma queda na tensão da corrente ou uma mudança de fase, que é definida como ângulo de fase (AF), ou ainda, como arco tangente da relação Xc e $\mathrm{R}^{3}$.

A relação geométrica entre $Z, R, X c$ e $A F$ depende da frequência da corrente elétrica administrada (Figura 1). Em baixas frequências (f1) $(\sim 1$ $\mathrm{kHz})$, a impedância das células e de suas membranas é muito alta para que a corrente consiga penetrar o conteúdo celular, então as membranas funcionam como resistores e apenas o fluido extracelular pode ser medido ${ }^{3,4}$. O componente capacitivo do sistema é um circuito aberto, sendo a Xc igual a zero, e a Z puramente resistiva $\left(R_{o}\right)^{15}$.

Conforme aumenta a frequência, a Xc aumenta na proporção da R, formando o AF. Nesse ponto máximo, o semicírculo atinge a frequência característica (fc) específica para o sistema. A fc é o valor máximo do semicírculo imaginário da bioimpedância e, ao alcançá-la, a Xc começa a diminuir na proporção da $R$, enquanto ocorre aumento na frequência da corrente elétrica ${ }^{15}$.

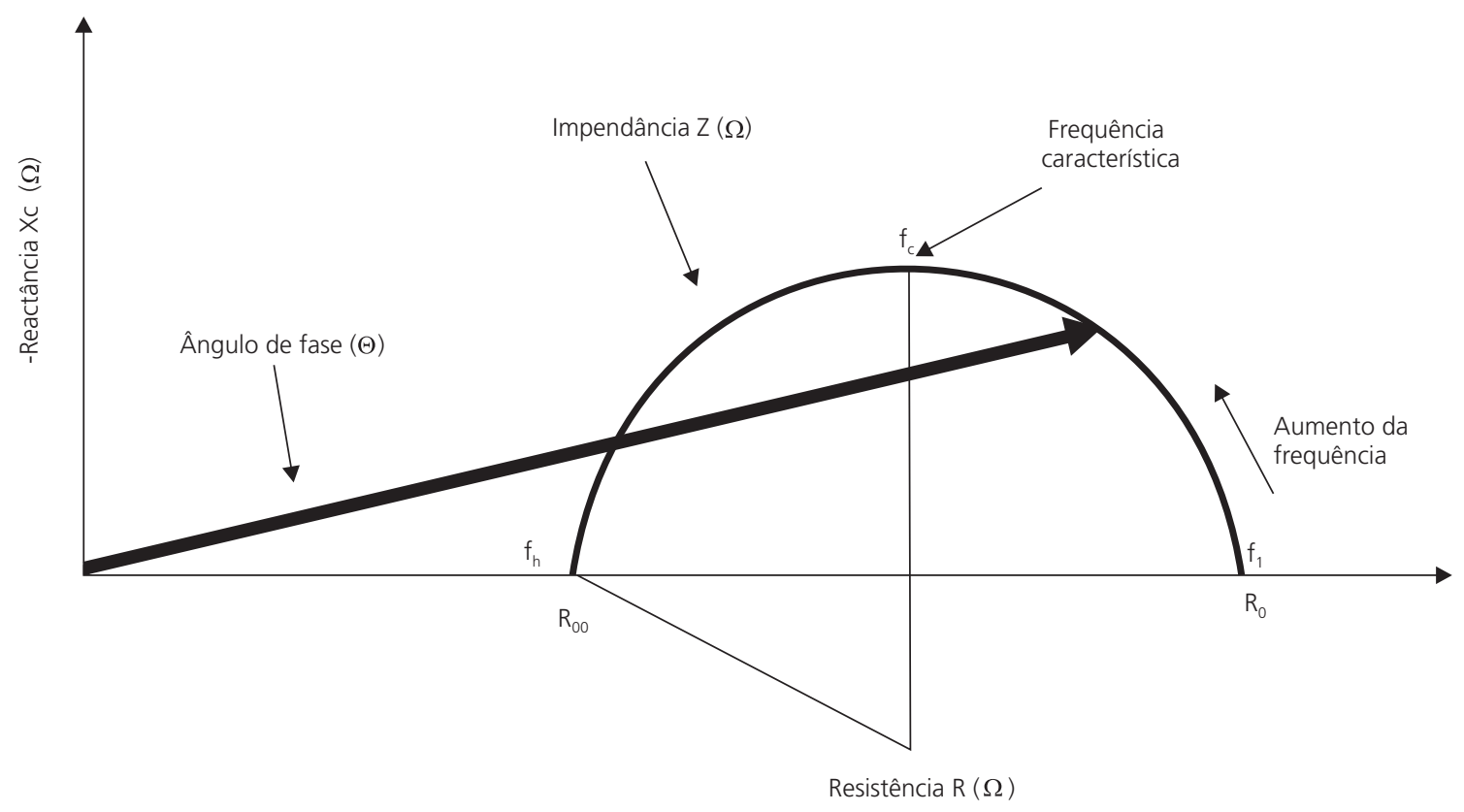

Figura 1. Demonstração da derivação gráfica do ângulo de fase, sua relação com a resistência, reactância, impedância e frequência da corrente aplicada através do Gráfico de Cole.

Fonte: Adaptado de Cole $^{16}$ 
Em frequências maiores (fh) (50 kFz e 100 $\mathrm{kHz}$ ), a corrente elétrica passa através das membranas celulares, permitindo as medidas de impedância dentro e fora das células, determinando o balanço hídrico intra e extracelular, causando redução na $\mathrm{Xc}$, aumento na $\mathrm{R}$ e diminuição do $\mathrm{AF}^{3,4}$. Em altas frequências, o componente capacitivo do sistema é um curto-circuito, sendo a $Z$ novamente puramente resistiva $\left(R_{o 0}\right)^{15}$.

Dessa forma, é possível determinar a relação entre $Z$ e os compartimentos corporais, onde a magnitude da $Z$ é igual ao vetor função de $R$ e $X c$, ou seja, $Z=R^{2}+X c^{2}$, que é dependente da frequência ${ }^{3}$.

Assim, através dos valores obtidos para essas variáveis ( $Z, R$ e Xc), em diferentes frequências, o analisador calcula a quantidade de água corporal total e sua distribuição intra e extracelular e, assumindo uma hidratação constante, determina primeiramente a massa corporal magra e, logo, a composição corporal3,15.

O gráfico de Cole (Figura 1) permite visualizar essas descrições. O gráfico fundamenta-se na aproximação dos valores obtidos por uma curva e no prolongamento desta até o eixo $R$, formando um semicírculo. Esse prolongamento permite extrapolar valores de $Z$ obtidos para cada frequência (entre $5 \mathrm{kHz}$ e $1.000 \mathrm{kHz}$ ), relacionando $\mathrm{Z}, \mathrm{R}, \mathrm{Xc}$, $A F$ e frequência. Salienta-se que esta é uma abordagem teórica, uma vez que frequências muito baixas ou muito altas não devem ser introduzidas no corpo, devido à ocorrência de múltiplas dispersões ou ainda, ao possível estímulo elétrico de alguns tecidos condutores, como o tecido cardíaco 3,8

\section{$O$ método da BIA}

Uma variedade de aparelhos de BIA tornou-se comercialmente disponível a partir da década de 1990, e hoje é possível encontrar aqueles que avaliam a composição corporal de forma tradicional e segmentar ${ }^{3}$.

Quanto ao tipo de frequência, atualmente é possível encontrar aparelhos monofrequenciais ou de frequência única (50 kHz), os mais usados, e aparelhos multifrequenciais, com frequência de 5 a $1.000 \mathrm{kHz}^{5}$.

A transmissão da corrente elétrica pelo corpo dá-se, geralmente, por quatro sensores metálicos (modelo tetrapolar) que, em contato com as mãos e/ou pés, registram a impedância dos segmentos corporais entre os membros superiores e o tronco, ou somente entre os membros inferiores, ou ainda entre os membros superiores e os inferiores ${ }^{17,18}$.

O aparelho que avalia os segmentos superiores e os inferiores é o modelo mais utilizado da BIA. Consiste no emprego de quatro eletrodos fixados no hemicorpo direito do indivíduo avaliado: na mão, próximos à articulação metacarpo-falângea da superfície dorsal; no pulso, entre as proeminências distais do rádio e da ulna; no pé, no arco transverso da superfície superior; e no tornozelo, entre os maléolos medial e lateral3,4,19.

Uma corrente de excitação é aplicada aos eletrodos-fonte (distais) na mão e no pé, e a queda de tensão, provocada pela impedância, é detectada pelo eletrodo-sensor (proximal) localizado no pulso e no tornozelo ${ }^{4}$. Sua análise baseia-se na medida da $\mathrm{R}$ total do corpo à passagem de uma corrente elétrica de baixa amplitude $(800 \mu \mathrm{A})$ e alta frequência $(50 \mathrm{kHz})^{4,17}$.

Em 2004, a BIA segmentar foi desenvolvida. Ela aborda aspectos da análise da composição corporal que podem eliminar certas inconsistências existentes na avaliação da massa corporal total, analisando o corpo por segmento, ou seja, essa técnica determina isoladamente a massa dos membros e do tronco ${ }^{3}$.

Estudiosos afirmam que as alterações na massa magra do tronco, separadamente, provavelmente não são descritas de forma confiável através das medidas de impedância do corpo inteiro e, ainda, que mudanças na impedância do corpo inteiro podem estar relacionadas às alterações tanto na massa magra dos membros como do tronco ${ }^{3,20}$, donde a importância da avaliação isolada. 
A BIA segmentar tem sido utilizada para determinar deslocamentos e distribuição de fluidos em algumas doenças, como ascite e insuficiência renal, e em cirurgias. Essa técnica pode também ser útil para fornecer informações sobre o acúmulo de líquidos na região pulmonar e abdominal ${ }^{3}$.

O modelo segmentar é um método que merece consideração e até o presente não existem estudos conclusivos sobre sua aplicação. Assim, pesquisas são necessárias para examinar a acurácia desse método na avaliação da composição corporal e outras funções.

\section{Modelos de predição}

A partir dos valores da $\mathrm{R}$ e da Xc obtidos pela BIA, são utilizadas diferentes equações de regressão disponíveis na literatura, para estimar os componentes corporais, e assim, determinar os valores de massa de gordura, massa magra e água corporal. Essas equações preditivas são ajustadas para sexo, etnia, idade, peso, altura e nível de atividade física ${ }^{3-6,21}$.

Estudos de desenvolvimento e validação de equações de BIA vêm sendo amplamente realizados, e o emprego da BIA na avaliação da composição corporal tem-se tornado comum em circunstâncias clínicas diversas, embora existam algumas controvérsias sobre seu uso, principalmente em condições onde há uma alteração do estado de hidratação dos indivíduos 3,4,6,22. Assim, se um indivíduo apresentar hiper-hidratação, o valor da massa magra será superestimado, por ser através da quantidade de água corporal total que se obtém a massa corporal magra.

Segal et al. ${ }^{4}$ e Segal et al..$^{23}$ estudaram a massa magra de indivíduos obesos e não obesos, observando que a massa magra avaliada pela BIA em obesos foi superestimada quando comparada com a densitometria corporal, considerada método de referência. Assim, para uma melhor correlação, os autores determinam várias equações específicas para essa população.
Utilizando equações propostas por Segal et al. ${ }^{23}$ outros autores concluíram que estas, que consideram sexo, peso, altura e idade, produziram uma boa correlação entre a BIA e os métodos de referência para estimar a massa magra. Entretanto, em indivíduos obesos severos ( $>48 \%$ de gordura corporal), as equações podem provocar erros sistemáticos ${ }^{24}$.

Para a estimativa dos componentes corporais, a BIA estima primeiramente a quantidade de água corporal total e, para isso, pressupõe um grau estável de hidratação e de conteúdo mineral ósseo. A suposição não é válida para indivíduos obesos, considerando que estes possuem um maior nível de hidratação inerente à obesidade, ocorrendo uma subestimação de gordura corporal25.

Outro estudo, que avaliou 1829 pacientes (1 474 brancos e 355 negros), também desenvolveu equações para uso em estudos epidemiológicos, utilizando variáveis como sexo, estatura, resistência e peso corporal, com excelente acurácia para avaliar água corporal total e massa ma$\mathrm{gra}^{26}$.

É importante salientar que as equações de predição variam conforme o aparelho e apresentam validade apenas para a população de origem, o que constitui um fator limitante para sua utilização em outros grupos populacionais.

Em adição, ao considerar a importância da avaliação da composição corporal, especialmente em indivíduos obesos, a BIA pode ser utilizada para tal fim, porém essa aplicabilidade também requer a utilização de equações específicas no intuito de aumentar a confiabilidade da estimativa de gordura corporal em determinada população. Portanto, independentemente da população a ser avaliada, deve-se analisar com cuidado a escolha de uma equação de BIA que seja específica para determinado grupo de indivíduos $3,5,18,22$.

\section{BIA x gordura visceral}

Outra vertente de utilização da BIA é a estimativa de gordura abdominal. Encontram-se 
na literatura novas técnicas de referência para avaliar a obesidade central e a gordura visceral através da BIA. Essa técnica consiste no posicionamento dos eletrodos diretamente no abdômen ${ }^{27-29}$. Nesses estudos a BIA demonstrou forte correlação com a gordura abdominal e visceral, determinada por tomografia computadorizada e ressonância magnética, método que, segundo os autores, permite avaliar com mais precisão a gordura abdominal, incluindo a visceral. No entanto, esses estudos não são conclusivos quanto à validade da BIA para estimativa da gordura visceral.

Segundo seus fabricantes, o modelo da BIA segmentar inclui avaliação do nível de gordura visceral. Através da análise isolada do tronco, o método estima com mais precisão essa gordura. Contudo, devem ser desenvolvidas investigações para testar a confiabilidade desse modelo, pois não existem estudos na literatura que sustentem a afirmação.

\section{Aplicabilidade da BIA}

Apesar de a BIA ser indicada como um método preciso e confiável, discutem-se, na literatura, possíveis causas que dificultam o estabelecimento de um consenso acerca de seu uso, pois resultados obtidos em determinadas pesquisas revelam-se algumas vezes discrepantes. Dentre as possíveis razões, poderia ser mencionada a utilização de uma variabilidade de equações disponíveis para vários grupos de indivíduos, que são aplicadas de forma equivocada em amostras bastante heterogêneas. Além disso, podem interferir as diferenças étnicas e de composição corporal entre as populações, bem como o estado de hidratação dos indivíduos avaliados, como mencionado anteriormente. Dessa forma, pode-se afirmar que ainda pairam dúvidas quanto à precisão e à confiabilidade da técnica da BIA, quando comparada com outros recursos 1,5,6,18,22.

O Quadro 1 apresenta estudos que comparam a BIA com outros métodos de avaliação da composição corporal. Dentre os principais resultados encontrados por tais estudos, observou-se boa correlação da BIA com IMC, pregas cutâneas e circunferência da cintura, assim como com métodos de referência (pesagem hidrostática, absorção dos Raios $X$ de dupla energia) para estimar a composição corporal em diferentes amostras. Nota-se, entretanto, alta variabilidade nos achados, podendo isso ser atribuído às diferenças metodológicas quanto aos aparelhos e equações utilizados, às populações analisadas ${ }^{5}$, e ainda, aos métodos considerados "padrão-ouro", os quais também podem apresentar erros sistemáticos.

Outra observação importante diz respeito aos métodos estatísticos utilizados, sendo mais adequado analisar a concordância entre eles do que apenas sua correlação, uma vez que alguns indicadores podem apresentar alta correlação, mas não concordarem. Essas comparações devem empregar, de preferência, métodos de referência.

Tendo em vista que seus resultados podem ser afetados por diversas condições, o controle prévio de alguns fatores deve ser realizado para a confiabilidade do método da BIA na prática clínica ${ }^{4,18,30}$. São eles: calibração do aparelho, realizada regularmente; manutenção dos eletrodos em sacos fechados e protegidos do calor; posição do indivíduo avaliado, conforme recomendação do fabricante; jejum de 4 horas, antes do exame; abstinência alcoólica de 8 horas, antes do exame; abstinência de atividade física e sauna, por 8 horas, antes do exame; esvaziamento da bexiga antes da realização do exame; temperatura do ambiente em torno de $22^{\circ} \mathrm{C}$; pele sem lesões e limpa com álcool; distância entre os eletrodos de, no mínimo, 5cm; observância do ciclo menstrual; presença de obesidade; utilização de material isolante, como toalha entre as pernas; impedimento de contato com superfície metálica; vedação do procedimento para portadores de marca-passo.

\section{Ângulo de fase da BIA}

Através da BIA, o AF pode ser obtido por meio da relação entre medidas diretas da $\mathrm{R}$ e da 
Quadro1. Comparação da BIA com outros métodos de avaliação do estado nutricional.

\begin{tabular}{|c|c|c|c|}
\hline Autores & Amostra & Aparelhos utilizados & Resultados encontrados \\
\hline Franz $^{31}$ & $\begin{array}{l}125 \text { pacientes de } 44 \text { a } 80 \text { anos } \\
\text { de idade. }\end{array}$ & BIA 101Q-RJL & $\begin{array}{l}\text { Correlação positiva para valores de BIA, IMC } \\
\text { e PCT. }\end{array}$ \\
\hline Heitmann 32 & $\begin{array}{l}139 \text { dinamarqueses com ida- } \\
\text { de entre } 35 \text { e } 65 \text { anos. }\end{array}$ & BIA 101Q-RJL & $\begin{array}{l}\text { Estimativas confiáveis do IMC, } \Sigma \text { quatro do- } \\
\text { bras cutâneas e BIA para gordura corporal to- } \\
\text { tal. }\end{array}$ \\
\hline Rodrigues et al. ${ }^{5}$ & $\begin{array}{l}25 \text { homens brancos com ida- } \\
\text { de de } 18 \text { a } 36 \text { anos. }\end{array}$ & $\begin{array}{l}\text { RJL-101, Byodinamics } \\
\text { A-310,Maltron BF-900, } \\
\text { BF-906 }\end{array}$ & $\begin{array}{l}\text { A técnica de dobras cutâneas apresentou } \\
\text { maior correlação com a PH, quando compara- } \\
\text { da aos diferentes modelos de BIA. }\end{array}$ \\
\hline Barbosaet al. ${ }^{1}$ & $\begin{array}{l}20 \text { mulheres com idade entre } \\
62 \text { e } 79 \text { anos. }\end{array}$ & BIA 101Q-RJL & $\begin{array}{l}\text { Entre a BIA, as dobras cutâneas e DEXA, ne- } \\
\text { nhuma técnica deve ser aceita como método } \\
\text { único de referência nessa população; com- } \\
\text { parações podem ser úteis na interpretação dos } \\
\text { resultados. }\end{array}$ \\
\hline Rech et al. ${ }^{33}$ & $\begin{array}{l}15 \text { mulheres pós-menopausa, } \\
\text { saudáveis, praticantes de ati- } \\
\text { vidades aquáticas. }\end{array}$ & $\begin{array}{l}\text { Biodinamics } 310 \text { e ONROM } \\
\text { modelo HBF-300 }\end{array}$ & $\begin{array}{l}\text { A BIA bipolar foi o único método que apre- } \\
\text { sentou validade cruzada, tanto para a esti- } \\
\text { mativa da massa gorda, quanto livre de gor- } \\
\text { dura, quando comparado às dobras cutâneas } \\
\text { DEXA e BIA tetrapolar. }\end{array}$ \\
\hline Fernandez et al. ${ }^{34}$ & $\begin{array}{l}811 \text { jovens de } 11 \text { a } 17 \text { anos de } \\
\text { idade. }\end{array}$ & BIA 101Q-RJL & $\begin{array}{l}\text { BIA apresentou bom desempenho na identifi- } \\
\text { cação do excesso de gordura visceral e subcu- } \\
\text { tânea, em comparação com PCT e CC. }\end{array}$ \\
\hline Barreto-Silva et al. ${ }^{25}$ & $\begin{array}{l}105 \text { pacientes com doença re- } \\
\text { nal crônica, estágios } 3 \text { e } 4 \text {. }\end{array}$ & Biodinamics 310 & $\begin{array}{l}\text { Para não obesos, o IMC, as dobras cutâneas } \\
\text { e a BIA podem ser aplicados com boa equiva- } \\
\text { lência para estimar gordura corporal. }\end{array}$ \\
\hline Rodríguez et al. ${ }^{35}$ & $\begin{array}{l}230 \text { pré-escolares argentinas } \\
\text { saudáveis, de } 4 \text { a } 6 \text { anos. }\end{array}$ & BIA Maltron BF-900 & $\begin{array}{l}\text { Alta correlação entre IMC, CC, BIA e DEXA } \\
\text { para estimar composição corporal. }\end{array}$ \\
\hline Lima et al. ${ }^{21}$ & $\begin{array}{l}60 \text { homens idosos, de } 60 \text { a } 81 \\
\text { anos de idade. }\end{array}$ & Biodinamics - BF-310 & $\begin{array}{l}\text { Forte correlação entre BIA, DEXA e IMC } \\
(r=0,90) \text {. }\end{array}$ \\
\hline Cocetti et al. ${ }^{36}$ & 1286 escolares de 7 a 9 anos. & TANITA TBF-300 ${ }^{\mathrm{a}}$ & $\begin{array}{l}\text { Alta e significante correlação entre dobras } \\
\text { cutâneas e BIA para o percentual de gordura } \\
\text { corporal, para massa gorda e massa magra } \\
\text { mostrando boa concordância. }\end{array}$ \\
\hline
\end{tabular}

BIA: bioimpedância elétrica; IMC: índice de massa corporal; PCT: prega cutânea triciptal; $\Sigma$ - somatório; PH: pesagem hidrostática: DEXA: absorção do raios $X$ de dupla energia; CC: circunferência da cintura.

$X c$, sendo calculado diretamente pela equação $X \mathrm{C} / \mathrm{R} \times 180^{\circ} / \Pi^{4,11,12,37}$. A variação do AF ocorre entre zero grau (sistema sem membranas celulares, apenas resistivo) e 90 graus (sistema sem fluidos, apenas capacitivo), sendo que num indivíduo saudável o AF pode apresentar valores entre $4 \mathrm{e}$ 10 graus. Também se encontra na literatura que esse valor pode variar de 5 a 15 graus $^{4,6,38}$.

Esse ângulo é dependente da capacitância dos tecidos e está associado com a qualidade, tamanho e integridade celular. Trata-se de uma ferramenta de diagnóstico nutricional cada vez mais utilizada na prática clínica. Estudos recentes têm validado esse ângulo como indicador prognóstico em pacientes críticos $12,13,15,37,39$. Salienta-se que o $A F$, relacionado com o equilíbrio celular, tem sido utilizado como medida de gravidade de doença, como instrumento de avaliação funcional e como indicador geral de saúde ${ }^{40}$.

Portanto, variação no AF indica alterações na composição corporal, na função da membrana celular ou no estado de saúde. Valores de AF menores representam baixa Xc e alta $R$, e podem ser associados à existência ou agravamento de 
doença, a morte celular, ou a alguma alteração na permeabilidade seletiva da membrana. De outro lado, valores mais altos representam alta $X c$ e baixa $R$, podendo associar-se à maior quantidade de membranas celulares intactas, ou seja, maior massa celular corpórea, e a um adequado estado de saúde $12,13,37,39,41$.

Dessa forma, modificações na massa celular corporal ou defeitos funcionais das membranas celulares podem resultar em mudanças no AF. Assim, é esperado que ocorram mudanças em seus valores, de acordo com o sexo e o envelhecimento, pois com o passar dos anos a capacitância dos tecidos - associada à variabilidade do tamanho das células, à permeabilidade da membrana celular e à composição intracelular - torna-se diferente, assim como a distribuição dos fluidos corporais entre os tecidos ${ }^{6,42,43}$.

A grande vantagem desse parâmetro é que ele independe de equações de regressão e pode ser realizado mesmo em situações nas quais as concepções da BIA não são válidas para estimar a composição corporal ${ }^{6,11,42}$, eliminando uma enorme fonte de erro casual ${ }^{11}$. Esse parâmetro pode, inclusive, ser utilizado mesmo em pacientes cujo peso e altura não podem ser mensurados ${ }^{6,42}$, ampliando a aplicabilidade da BIA na prática clínica.

\section{AF como indicador prognóstico}

Pesquisas realizadas para investigar o papel do AF como indicador prognóstico em pacientes hospitalizados, em portadores de doenças graves (como alguns tipos de câncer, Human Immunodeficiency Virus (HIV), Síndrome de Imunodeficiência Adquirida (AIDS), insuficiência renal crônica, doença pulmonar obstrutiva crônica, cirrose hepática, bacteriemia) e em pacientes criticamente doentes, evidenciaram associação positiva entre os valores do AF e o tempo de sobrevida dos pacientes. Os autores sugerem que o AF poderia ser uma importante ferramenta para avaliar sinais clínicos e monitorar a progressão da doença, sendo até mesmo superior a outros indicadores séricos ou antropométricos 9, 12,13,15,37,39.
Em pacientes críticos, ainda, Barbosa-Silva et al. ${ }^{4}$ verificaram que o AF parece ser um importante fator prognóstico de complicações pós-operatórias, depois de ajustados os fatores de confusão, demonstrando que o método é útil na identificação de pacientes que necessitam de terapia nutricional.

\section{AF como indicador de estado nutricional}

O AF também tem sido utilizado como um indicador do estado nutricional. Especula-se que seja um marcador de nutrição relevante clinicamente, que possa caracterizar o acréscimo de massa extracelular corporal e o decréscimo de massa celular corporal; provavelmente, porque a nutrição está interligada tanto às alterações na integridade da membrana celular quanto às alterações no equilíbrio dos fluidos corporais $6,9,37,39,42-44$.

Ellis ${ }^{45}$, avaliando pacientes renais, observou que o AF tipicamente $<5^{\circ}$ pode ser interpretado como um indicador de expansão de água para o espaço extracelular e de redução da água intracelular.

A restrita utilização da BIA na prática clínica e em situações epidemiológicas deve-se à carência de valores de referência para a população. Destaca-se que tais valores são necessários para avaliar corretamente desvios individuais em relação à média populacional e também para comparar estudos ${ }^{6,12}$.

Apesar de o AF ser destacado como importante marcador de morbidade e mortalidade em uma série de doenças (e assim, um potencial indicador do estado nutricional $6,11-13,15,37,43,44)$, faz-se necessário estudá-lo com maior profundidade, pois ainda é um parâmetro pouco utilizado e existem controvérsias sobre sua relação com os marcadores de estado nutricional. No Quadro 2 estão apresentados diferentes estudos que utilizaram o AF, podendo inclusive exercer um papel complementar aos indicadores habitualmente utilizados na prática clínica. 
Quadro 2. Avaliação do AF como indicador do estado nutricional.

\begin{tabular}{|c|c|c|}
\hline Autores & Amostra & Resultados encontrados \\
\hline Maggiori ${ }^{44}$ & 131 pacientes em hemodiálise. & $\begin{array}{l}\text { AF apresentou correlação significativa com todos os índices } \\
\text { nutricionais, exceto IMC. }\end{array}$ \\
\hline Barbosa-Silva et al. ${ }^{42}$ & $\begin{array}{l}279 \text { pacientes de cirurgia gastrointestinal } \\
\text { eletiva. }\end{array}$ & $\begin{array}{l}\text { Concordância moderada entre a avaliação subjetiva global e o AF. } \\
\text { Pacientes desnutridos graves tiveram menores valores de AF. }\end{array}$ \\
\hline Mushnick et al. ${ }^{46}$ & $\begin{array}{l}48 \text { pacientes em diálise peritonial, com } \\
\text { idade média de } 51, \mathrm{DP}=15 \text { anos. }\end{array}$ & $\begin{array}{l}\text { A R correlacionou-se a peso corporal, IMC e massa celular corpo- } \\
\text { ral. A Xc à pré-albumina e albumina; e o AF com a massa celular } \\
\text { corporal, pré-albumina e albumina. }\end{array}$ \\
\hline De Luis et al. ${ }^{47}$ & $\begin{array}{l}100 \text { homens com } \mathrm{HIV}^{+} \text {, com idade mé- } \\
\text { dia de } 39,1, \mathrm{DP}=9,9 \text { anos. }\end{array}$ & $\begin{array}{l}\text { O peso corporal, IMC, transferrina e somatomedina } C \text { foram maiores } \\
\text { no grupo com } A F \geq 8,2 \text { (AF médio). }\end{array}$ \\
\hline Gupta et al. ${ }^{9}$ & $\begin{array}{l}58 \text { pacientes com câncer pancreático - } \\
\text { estágio IV. }\end{array}$ & $\begin{array}{l}\text { Significativa correlação positiva entre o AF e a albumina e a pré- } \\
\text {-albumina. }\end{array}$ \\
\hline Barbosa-Silva et al. ${ }^{6}$ & $\begin{array}{l}1967 \text { americanos saudáveis, de } 18 \text { a } 94 \\
\text { anos de idade. }\end{array}$ & $\begin{array}{l}\text { O AF muda conforme o sexo, idade, IMC e percentual de gordura } \\
\text { corporal. Ainda, o AF demonstrou correlação positiva com o IMC. }\end{array}$ \\
\hline Hengsterman et al. ${ }^{48}$ & $\begin{array}{l}484 \text { pacientes com multimorbidades, } \\
\text { com idade média de } 79,6, \mathrm{DP}=7,6 \text { anos. }\end{array}$ & $\begin{array}{l}\text { O IMC reduziu significativamente nos pacientes com úlcera de pres- } \\
\text { são (UP). A BIA não apresentou resultados significantes para R e Xc, } \\
\text { mas para AF na UP. }\end{array}$ \\
\hline Azevedo et al. ${ }^{41}$ & 75 pacientes, sendo 65 com sepse. & $\begin{array}{l}\text { Tendência de associação entre o AF e escore prognóstico padrão } \\
\text { para avaliação da gravidade de doença, evolução, disfunção de } \\
\text { múltiplos órgãos e sistemas, e tempo de internação. }\end{array}$ \\
\hline Gupta et al. ${ }^{37}$ & $\begin{array}{l}73 \text { pacientes portadores de câncer co- } \\
\text { lorretal - estágios III e IV. }\end{array}$ & $\begin{array}{l}\text { Pacientes bem nutridos apresentaram média de AF significativa- } \\
\text { mente maior que os desnutridos. O AF pode ser um potencial indi- } \\
\text { cador nutricional. }\end{array}$ \\
\hline Sonsin et al. ${ }^{49}$ & 30 pacientes com disfagia orofaríngea. & $\begin{array}{l}\text { Os pacientes com } \mathrm{AF} \geq 4^{\circ} \text { apresentaram perspectivas de recupera- } \\
\text { ção nutricional e clínica. }\end{array}$ \\
\hline
\end{tabular}

AF: ângulo de fase; IMC: índice de massa corporal; R: resistência; Xc: reactância; UP: úlcera de pressão; DP: desvio-padrão.

\section{ONCLUSÃO}

Os estudos não só revelam uma importante habilidade da BIA em determinar o fracionamento dos componentes corporais, mas também valorizam sua utilização como marcador de estado nutricional e de avaliação de dano celular. Entretanto, é recomendado o estabelecimento de critérios mais confiáveis para análise e interpretação dos resultados. Nesse sentido, devem ser priorizadas pesquisas que desenvolvam equações específicas para a população brasileira, inclusive para diferentes grupos etários.

\section{COLABORADORES}

M. EICKEMBERG foi responsável pelo levantamento bibliográfico e redação do artigo. C.C. OLIVEIRA e A.K.C. RORIZ contribuíram na redação e revisão do texto. L.R. SAMPAIO responsabilizou-se pela orientação e coordenação do trabalho.

\section{REFERÊ NCIAS}

1. Barbosa AR, Santarem JM, Jacob Filho W, Meirelles ES, Marucci MFN. Comparação da gordura corporal de mulheres idosas segundo antropometria, bioimpedância e DEXA. Arch Latinoam Nutr. 2001; 51(1):49-56.

2. Janssen I, Baumgartner RN, Ross R, Rosenberg $I H$, Roubenoff R. Skeletal muscle cutpoints associated with elevated physical disability risk in older men and women. Am J Epidemiol. 2004; 159(4):13-21.

3. Kyle UG, Bosaeus I, Lorenzo AD, Deurenberg P, Elia M, Gómez JM, et al. Bioelectrical impedance analysis - part I: review of principles and methods. Clin Nutr. 2004; 23:1226-46.

4. Britto EP, Mesquita ET. Bioimpedância elétrica aplicada à insuficiência cardíaca. Rev SOCERJ. 2008; 21(3):178-83. 
5. Rodrigues MN, Silva SC, Monteiro WD, Farinatti PTV. Estimativa da gordura corporal através de equipamentos de bioimpedância, dobras cutâneas e pesagem hidrostática. Rev Bras Med Esporte. 2001; 7(4):125-131.

6. Barbosa-Silva MC, Barros AJ, Wang J, Heymsfield $\mathrm{SB}$, Pierson RN. Bioelectrical impedance analysis: population reference values for phase angle by age and sex. Am J Clin Nutr. 2005; 82(1):49- 52.

7. Atzler E, Lehmann G. Über ein Neues Verfahren zur Darstellung der Herztätigkeit (Dielektrographie). Arbeitsph. 1932; 5(6):636-80.

8. Nyboer J, Hannapel L. Electrical impedance plethysmography: a physical and physiologic approach to peripheral vascular study. Circulation. 1950; 2(6):811-21. doi: 10.1161/01.CIR.2.6.811.

9. Thomasset A. Bioeletrical properties of tissue impedance measurements. Lyon Med.1962; 207: 107-18.

10. Hoffer EC, Meador CK, Simpson DC. Correlatiion of whole-body impedance with total body water volume. J Appl Physiol. 1969; 27(4):531-4.

11. Gupta D, Lammersfeld CA, Burrows JL, Dahlk SL, Vashi PG, Grutsch JF, et al. Bioelectrical impedance phase angle in clinical practice: implications for prognosis in advanced colorectal cancer. Am J Clin Nutr. 2004; 80(6):134-38.

12. Gupta D, Lammersfeld CA, Vashi PG, King J, Dahlk $\mathrm{SL}$, Grutsch JF, et al. Bioelectrical impedance phase angle as a prognostic indicator in breast cancer. BMC Cancer. 2008; 8(1):249.

13. Gupta D, Lammersfeld CA, Vashi PG, King J, Dahlk $\mathrm{SL}$, Grutsch JF, et al. Bioelectrical impedance phase angle in clinical practice: implications for prognosis in stage IIIB and IV non-small cell lung cancer. BMC Câncer. 2009; 9:37.

14. Kamimura MA, Draibe AS, Sigulen DM, Cuppari L. Métodos de avaliação da composição corporal em pacientes submetidos à hemodiálise. Rev Nutr. 2004; 17(1):97-105. doi:10.1590/S1415-5273200 400010001.

15. Baumgartner RN, Chumlea WC, Roche AF. Bioelectric impedance phase angle and body composition. Am J Clin Nutr. 1988; 48(1):16-23.

16. Cole KS. Permeability and impermeability off cell membranes for íons. Quantitat Biol. 1940; 8(1): 110-22.

17. Heyward VH, Stolarczyk LM. Avaliação da composição corporal aplicada. São Paulo: Manole; 2000.

18. Jambassi Filho JC, Cyrino ES, Gurjão ALD, Braz IA, Gonçalves R, Gobbi S. Estimativa da composição corporal e análise de concordância entre analisa- dores de impedância bioelétrica bipolar e tetrapolar. Rev Bras Med Esporte. 2010; 16(1):13-7.

19. Marques MB, Heyward V, Paiva CE. Validação cruzada de equações de bio-impedância em mulheres brasileiras por meio de absortometria radiológica de dupla energia (DXA). Rev Bras Ciênc Mov. 2000; 8(4):14-20.

20. Pirlich M, Schutz T, Spachos T, Ertl S, Weiss ML, Lochs $\mathrm{H}$, et al. Bioelectrical impedance analysis is a useful bedside technique to assess malnutrition in cirrhotic patients with and without ascites. Hepatology. 2000; 32(6):1208-15.

21. Lima LRA, Rech CR, Petroski EL. Utilização da impedância bioelétrica para estimativa da massa muscular esquelética em homens idosos. Arch Latinoam Nutr. 2008; 58(4):386-91.

22. Rezende F, Rosado L, Franceschini S, Rosado G, Ribeiro R, Marins JCB. Revisão crítica dos métodos disponíveis para avaliar a composição corporal em grandes estudos populacionais e clínicos. Arch Latinoam Nutr. 2007; 57(4):327-34.

23. Segal KR, Gutin B, Presta, Wang J, van Itallie TB. Estimation of human body composition by electrical impedance methods a comparative study. J Appl Physiol. 1985; 58(5):1565-71.

24. Gray D, Bray G, Gemayel N, Kaplan K. Effect of obesity on bioelectrical impedance. Am J Clin Nutr. 1989; 50(2):255-60.

25. Barreto-Silva MI, Avesani CM, Vale B, Lemos C, Bregman R. Agreement between anthropometry and bioelectrical impedance for measuring body fat in nonobese and obese nondialyzed chronic kidney disease patients. J Renal Nutr. 2008; 18(4): 355-62.

26. Sun SS, Chumlea WC, Heymsfield SB, Lukaski HC, Schoeller D, friedl K, et al. Development of bioelectrical impedance analysis prediction equations for body composition with the use of a multicomponent model for use in epidemiologic surveys. Am J Clin Nutr. 2003; 77(2):331-40.

27. Nagai $M$, Komiya $H$, Mori $Y$, Otha $T$, Kasahara $Y$, Ikeda $Y$. Development of a new method for estimating visceral fat área with multi frequency bioelectrical impedance. J Exp Med. 2008; 214(2): 105-12.

28. Watson S, Blundell HL, Evans WD, Griffiths $H$, Newcombe RG, Rees DA. Can abdominal bioelectrical impedance refine the determination of visceral fat from waist circumference? Physiol Meas. 2009; 30(7):N53-8.

29. Ryo M, Maeda K, Onda T, katashima M, Okumiya $A$, Nishida $M$, et al. A new simple method for the measurement of visceral fat accumulation by bioelectrical impedance. Diab Care. 2005; 28(2): 451-3. 
30. Sant'anna MSL, Tinoco ALA, Rosado LEFPL, Sant'anna LFR, Mello AC, Brito ISS, et al. Body fat assessment by bioelectrical impedance and its correlation with different anatomical sites used in the measurement of waist circumference in children. J Pediatr. 2009; 85(1):61-6.

31. Franz LBB. Bioimpedância elétrica como método de avaliação da composição corporal de indivíduos adultos e idosos. ljuí: Unijuí; 1998.

32. Heitmann BL. Avaliação de gordura corporal estimado do índice de massa corporal, dobras cutâneas e impedância: um estudo comparativo. Eur J Clin Nutr. 1990; 44(11):831-7.

33. Rech CR, Silva AT, Lunardi CC, Bohrer T, Petroski EL. Comparação da absormetria radiológica de dupla energia, antropometria e impedância bioelétrica na avaliação da composição corporal em mulheres. Rev Dig Buenos Aires. 2005 [acesso 2010]; 10(91). Disponível em: <http://www.efde portes.com/efd91/antrop.htm>.

34. Fernandez RA, Rosa CSC, Buonani C, Oliveira AR, Freitas Junior IF. Utilização da impedância bioelétrica na indicação do excesso de gordura visceral e subcutânea. J Pediatr. 2007; 83(6):529-34.

35. Rodríguez PN, Bermúdez EF, Rodríguez GS, Spina MA, Zeni SN, Friedman SM, et al. Composición corporal en niños preescolares: comparación entre métodos antropométricos simples, bioimpedancia y absorciometría de doble haz de rayos X. Arch Argent Pediantr. 2008; 106(2):102-9.

36. Cocetti M, Castilho SD, Barros Filho, AA. Dobras cutâneas e bioimpedância elétrica perna-perna na avaliação da composição corporal de crianças. Rev Nutr. 2009; 22(4):527-36. doi: 10.1590/S1415-527 32009000400008 .

37. Gupta D, LIS CG, Dahlk SL, King J, Vashi PG, Grutsch JF, et al. The relationship between bioelectrical impedance phase angle and subjective global assessment in advanced colorectal cancer. Nutr J. 2008; 7:19. doi: 10.1186/1475-2891-7-19

38. Silva LMDL, Caruso L, Martini LA. Aplicação do ângulo de fase em situações clínicas. Rev Bras Nutr Clin. 2007; 22(4):317-21.

39. Selberg O, Selberg D. Norms and correlates of bioimpedance phase angle in healthy human subjects, hospitalized patients, and patients with liver cirrhosis. Eur J Appl Physiol. 2002; 86(6):509-16.

40. Barbosa DMO, Daltro CS, Torres AC, Reis GP, Costa GLOB, Costa MSG, et al. Aplicação clínica do ângulo de fase em oncologia. Rev Bras Nutr Clín. 2008; 23(3):209-42.

41. Azevedo ZMA, Silva DR, Dutra MVP, Elsas MICG, Barbosa-Silva MCG, Fonseca VM. Associação entre ângulo de fase, PRISM I e gravidade da sepse. Rev Bras Terap Intens. 2007; 19(3):297-303.

42. Barbosa-Silva MC, Barros AJ, Post CL, Waitzberg DL, Heymsfield SB. Can bioelectrical impedance analisys identify malnutrition in preoperative nutrition assessment? Nutrition. 2003; 19(5):422-6.

43. Scheunemann L, Wazlawik E, Trindade EBSM. Aplicação do ângulo de fase na prática clínica nutricional. Rev Bras Nutr Clín. 2008; 23(4):292-7.

44. Maggiore Q, Nigrelli S, Ciccarelli C, Grimaldi C, Rossi GA, Michelassi C. Nutritional and prognostic correlates of boielectrical impedance indexes in hemodialysis patients. Kidney Int. 1996; 50:2103-8.

45. Ellis KJ. Human body composition: in vivo methos. Physiol Rev. 2000; 80(2):649-80.

46. Mushnick R, Fein PA, Mittman N, Goel N, Chattopadhyay J, Avram MM. Relatinship of bioelectrical impedance parameters to nutrition and survival in peritoneal dialysis patients. Kidney Int. 2003; 64(S87):S53-6. doi: 10.1046/j-1523-1755.6 4.587.22.x.

47. De Luis Da, Aller R, Bachiller P, Gonzalez-Sagrado $\mathrm{M}$, Martin J, Izaola O. Influence of hormonal status and oral intake on phase angle in HIV-infected men. Nutrition. 2004; 20(9):731-4.

48. Hengstermann S, Fischer A, Steinhagen-Thiessen E, Schulz R. Nutrition status and pressure ulcer. What we need for nutrition screening. J Parent Ent Nutr. 2007; 31(4):288-94.

49. Sonsin PB, Bonfim C, Silva ALND, Caruso L. Análise da assistência nutricional a pacientes disfágicos hospitalizados na perspectiva de qualidade. Mundo Saúde S. Paulo. 2009; 33(3):310-9.

Recebido em: 3/8/2010

Versão final reapresentada em: 12/9/2011 Aprovado em: 21/9/2011 
